Pacific Journal of Mathematics

ISOMORPHISM INVARIANTS FOR ABELIAN GROUPS 


\title{
ISOMORPHISM INVARIANTS FOR ABELIAN GROUPS MODULO BOUNDED GROUPS
}

\author{
RONALD J. ENSEY
}

Let $\mathscr{A}$ be the category of Abelian groups, let $\mathscr{B}$ be the class of bounded Abelian groups, and form the quotient category $\mathscr{A} / \mathscr{B}$. The principal goal of this paper is a complete set of invariants for direct sums of countable reduced $p$-groups, such groups considered as objects of the category $\mathscr{A} / \mathscr{B}$. Specifically, it will be shown that two direct sums of countable reduced $p$-groups $G$ and $H$ are isomorphic in $\mathscr{A} / \mathscr{B}$ if and only if there is an integer $k \geqq 0$ such that for all ordinal numbers $\alpha$ and all integers $r \geqq 0$

and

$$
\sum_{j=0}^{r} f_{G}(\alpha+k+j) \leqq \sum_{j=0}^{r+2 k} f_{H}(\alpha+j)
$$

$$
\sum_{j=0}^{r} f_{H}(\alpha+k+j) \leqq \sum_{j=0}^{r+2 k} f_{G}(\alpha+j)
$$

where $f_{G}(\beta)$ and $f_{H}(\beta)$ denote the $\beta$ th Ulm invariants of $G$ and $H$, respectively. Thus a complete set of $\mathscr{A} / \mathscr{B}$-isomorphism invariants for such groups is an equivalence class of UIm invariants, the equivalence relation being given by these two inequalities.

The concept of quasi-isomorphism as defined by B. Jonsson [11] has come to play a significant role in the theory of torsion-free Abelian groups. However, nothing of significance had been done for quasi-isomorphism of torsion groups until R. Beaumont and R. Pierce [1] gave necessary and sufficient conditions on the Ulm invariants for two countable reduced $p$-groups to be quasi-isomorphic. This lack of results for $p$-groups was pointed out by E. A. Walker [14], who in fact submitted that the definition of quasi-isomorphism should be isomorphism in the category $\mathscr{A} / \mathscr{B}$. The importance of quasi-isomorphism for torsion-free groups motivates the study of Abelian groups as objects of $\mathscr{O} / \mathscr{B}$.

2. Preliminaries. The word group will always mean Abelian group, the category of Abelian groups will be denoted by $\mathscr{A}$, and the class of bounded Abelian groups by $\mathscr{B}$. The term Abelian category will be used in the sense of MacLane [13]. For $G$ and $H$ in an Abelian category $\mathscr{C}, \operatorname{Hom}_{\mathscr{C}}(G, H)$ will denote the group of maps from $G$ to $H . \operatorname{Hom}_{\mathscr{N}}(G, H)$ will be written simply as $\operatorname{Hom}(G, H)$. Any unexplained notation or terminology will conform with that of Fuchs [4] or MacLane [13]. 
A nonempty class $\zeta$ of $\mathscr{A}$ is a Serre class of $\mathscr{A}$ if for each exact sequence

$$
0 \longrightarrow G \longrightarrow H \longrightarrow K \longrightarrow 0
$$

of groups, $H$ is in $\zeta$ if and only if $G$ and $K$ are in $\zeta$. It is clear that $\mathscr{B}$ is a Serre class of $\mathscr{A}$, and it is the quotient category $\mathscr{A} / \mathscr{B}$ as defined by Grothendieck [6] with which this paper is concerned.

The objects of $\mathscr{A} / \mathscr{B}$ are just the objects of $\mathscr{A}$, and $\operatorname{Hom}_{\mathscr{A} / \mathscr{A}}(G, H)$ is the direct limit of the groups $\operatorname{Hom}\left(G^{\prime}, H / H^{\prime}\right)$, the limit being taken over all pairs $\left(G^{\prime}, H^{\prime}\right)$ with $G^{\prime} \subseteq G, H^{\prime} \subseteq H$, and $G / G^{\prime}, H^{\prime} \in \mathscr{S}$. To define composition let $\bar{f} \in \operatorname{Hom}_{\mathscr{A} \mid \mathscr{B}}(G, H)$ and $\bar{g} \in \operatorname{Hom}_{\mathscr{A} \mid \mathscr{A}}(H, K)$. Then $\bar{f}$ comes from an $f: G^{\prime} \rightarrow H / H^{\prime}$ and $\bar{g}$ comes from a $g: H^{\prime \prime} \rightarrow K / K^{\prime}$ where $G / G^{\prime}, H^{\prime}, H / H^{\prime \prime}, K^{\prime} \in \mathscr{\mathscr { B }}$. Let $G^{\prime \prime}=f^{-1}\left(\left(H^{\prime \prime}+H^{\prime}\right) / H^{\prime}\right)$. Then $G^{\prime} / G^{\prime \prime} \in \mathscr{B}$, and since

$$
0 \longrightarrow G^{\prime} / G^{\prime \prime} \longrightarrow G / G^{\prime \prime} \longrightarrow G / G^{\prime} \longrightarrow 0
$$

is exact, $G / G^{\prime \prime} \in \mathscr{B}$. Let $K^{\prime \prime} / K^{\prime}=g\left(H^{\prime} \cap H^{\prime \prime}\right)$. Then $K^{\prime \prime} \in \mathscr{B}$ since $H^{\prime}, K^{\prime} \in \mathscr{B}$. Now let $h$ be the composition

$$
G^{\prime \prime} \stackrel{f^{\prime}}{\longrightarrow}\left(H^{\prime \prime}+H^{\prime}\right) / H^{\prime} \simeq H^{\prime \prime} / H^{\prime} \cap H^{\prime \prime} \stackrel{g^{\prime}}{\longrightarrow} K / K^{\prime \prime}
$$

where $f^{\prime}$ is the restriction of $f$ to $G^{\prime \prime}$ and $g^{\prime}$ is induced by $g$. Since $G / G^{\prime \prime}, K^{\prime \prime} \in \mathscr{B}, h$ determines an element $\bar{h} \in \operatorname{Hom}_{\mathscr{\mathscr { O }}}(G, K)$. It is straightforward that $\bar{h}$ is uniquely determined by $\bar{f}$ and $\bar{g}$, and that $\mathscr{A} / \mathscr{B}$ is a category with the definition $\bar{h}=\bar{g} \circ \bar{f}$. That $\mathscr{A} / \mathscr{B}$ is an Abelian category can be found in [5].

Let $f: G^{\prime} \rightarrow H / H^{\prime}$ and $g: G^{\prime \prime} \rightarrow H / H^{\prime \prime}$ where $G / G^{\prime}, H^{\prime}, G / G^{\prime \prime}, H^{\prime \prime} \in \mathscr{B}$. Let $\bar{f}$ and $\bar{g}$ be the elements of $\operatorname{Hom}_{\mathscr{A} \mid \mathscr{S}}(G, H)$ determined by $f$ and $g$, respectively. The following facts are taken from [14].

2.1. $\bar{f}=0$ if and only if $\operatorname{Im} f \in \mathscr{B}$.

2.2. $\bar{f}$ is an epimorphism if and only if Coker $f \in \mathscr{B}$.

2.3. $\bar{f}$ is a monomorphism if and only if $\operatorname{Ker} f \in \mathscr{B}$.

2.4. $\bar{f}=\bar{g}$ if and only if there are subgroups $S \subseteq G$ and $T \subseteq H$ such that

(a) $S \subseteq G^{\prime} \cap G^{\prime \prime}$

(b) $G / S \in \mathscr{B}$,

(c ) $H^{\prime}+H^{\prime \prime} \subseteq T$,

(d) $T \in \mathscr{B}$,

(e) the maps from $S$ to $H / T$ induced by $\bar{f}$ and $\bar{g}$ are the same.

From 2.2 and 2.3 it follows that $G$ and $H$ are isomorphic in $\mathscr{A} \mid \mathscr{B}$ if and only if there exist subgroups $S$ and $A$ of $G$, and $T$ and $B$ of $H$ such that $S / A \simeq T / B$ in $\mathscr{A}$ and $G / S, A, H / T, B \in \mathscr{B}$.

Two groups $G$ and $H$ are quasi-isomorphic, and we write $G \dot{\simeq} H$, if there exist isomorphic subgroups $S \subseteq G$ and $T \subseteq H$ such that $G / S$ 
and $H / T$ are bounded. It is clear that in general quasi-isomorphism implies isomorphism in $\mathscr{A} / \mathscr{S}$ and that the two concepts are equivalent if we restrict ourselves to torsion-free groups.

3. Fundamentals of isomorphism in $\mathscr{A} / \mathscr{S}$.

THEOREM 3.1. The following are equivalent.

(i) $G \simeq H$ in $\mathscr{A} / \mathscr{B}$.

(ii) There exisis a bounded subgroup $B \subseteq H$ such that $G \dot{\sim} H / B$.

(iii) There exists a positive integer $k$ and homomorphisms $f: G \rightarrow H$ and $g: H \rightarrow G$ such that $g f=k$ and $f g=k$.

Proof. Suppose $G \simeq H$ in $\mathscr{A} / \mathscr{B}$. Then there is a homomorphism $f: G^{\prime} \rightarrow H / H^{\prime}$ such that $G / G^{\prime}, H^{\prime}$, Ker $f$, Coker $f$ are bounded. By 2.4, we can take $G^{\prime}=m G$ and $H^{\prime}=H[m]$ for some integer $m>0$. Let $n>0$ be a bound for $\operatorname{Ker} f$ and Coker $f$, and let $B / H[m]=$ $f((m G)[n])$. Let $\alpha$ be the composition

$$
m n G \longrightarrow(m G) /(m G)[n] \longrightarrow(H / H[m]) /(B / H[m]) \longrightarrow H / B
$$

where

$m n G \longrightarrow(m G) /(m G)[n]$ and $(H / H[m]) /(B / H[m]) \longrightarrow H / B$

are natural isomorphisms and

$$
(m G) /(m G)[n] \longrightarrow(H / H[m]) /(B / H[m])
$$

is the monomorphism induced by $f$. Now $\alpha$ is a monomorphism with cokernel bounded by $n$. Thus $G \dot{\simeq} H / B$ and since $B$ is bounded by $m n$, (i) implies (ii).

Suppose $G \dot{\simeq} H / B$ where $n B=0, n>0$. Then there exist isomorphic subgroups $S \subseteq G$ and $T / B \cong H / B$ such that $G / S$ and

$$
(H / B) /(T / B) \simeq H / T
$$

are bounded. Let $m>0$ with $m G \leqq S$ and $m H \subseteq T$, and let $\theta$ be the isomorphism $S \simeq T / B$. Let $f$ be the composition

$$
G \stackrel{m}{\longrightarrow} m G \leqq S \stackrel{\theta}{\longrightarrow} T / B \leqq H / B \stackrel{\pi_{1}}{\longrightarrow} H / H[n] \stackrel{\alpha}{\longrightarrow} n H \leqq H
$$

where $H / B \stackrel{\pi_{1}}{\longrightarrow} H / H[n]$ is the projection induced by $B \cong H[n]$ and $H / H[n] \stackrel{\alpha}{\longrightarrow} n H$ is the natural isomorphism. Let $g$ be the composition

$$
H \stackrel{\pi_{2}}{\longrightarrow} H / B \stackrel{m}{\longrightarrow}(m H+B) / B \subseteq T / B \stackrel{\theta^{-1}}{\longrightarrow} S \subseteq G
$$

where $H \stackrel{\pi_{2}}{\longrightarrow} H / B$ is the natural projection. Let $x \in G$ with $\theta(m x)=$ 
$y+B$. Then

$$
\begin{aligned}
x \stackrel{m}{\longrightarrow} m x \stackrel{\theta}{\longrightarrow} y+B \stackrel{\pi_{1}}{\longrightarrow} y+H[n] \stackrel{\alpha}{\longrightarrow} n y \\
\stackrel{\pi_{2}}{\longrightarrow} n y+B \stackrel{m}{\longrightarrow} m n y+B \stackrel{\theta^{-1}}{\longrightarrow} m^{2} n y .
\end{aligned}
$$

Let $y \in H$ with $\theta(x)=m y+B$. Then

$$
\begin{aligned}
y \stackrel{\pi_{2}}{\longrightarrow} y+B \stackrel{m}{\longrightarrow} m y+B \stackrel{\theta^{-1}}{\longrightarrow} x \stackrel{m}{\longrightarrow} m x \\
\stackrel{\theta}{\longrightarrow} m^{2} y+B \stackrel{\pi_{1}}{\longrightarrow} m^{2} y+H[n] \stackrel{\alpha}{\longrightarrow} m^{2} n y .
\end{aligned}
$$

Thus $g f=m^{2} n$ and $f g=m^{2} n$. Hence (ii) implies (iii).

Suppose there exists a positive integer $k$ and homomorphisms $f: G \rightarrow H$ and $g: H \rightarrow G$ such that $f g=k$ and $g f=k$. Since $g f=k$, Ker $f$ is bounded by $k$ and since $f g=k$, Coker $f$ is bounded by $k$. Hence $G \cong H$ in $\mathscr{O} / \mathscr{S}$ and (iii) implies (i).

COROLlaRY 3.2. Let $S$ and $T$ be subgroups of $G$ and $H$, respectively, such that $f(S) \subseteq T$ and $g(T) \subseteq S$ whenever $f: G \rightarrow H$ and $g: H \rightarrow G$. Let $G \simeq H$ in $\mathscr{A} / \mathscr{B}$. Then $S \simeq T$ in $\mathscr{A} / \mathscr{B}$ and $G / S \simeq H / T$ in $\mathscr{A} / \mathscr{B}$.

Proof. There exists an integer $k>0$ and homomorphisms $f: G \rightarrow H$ and $g: H \rightarrow G$ such that $g f=k$ and $f g=k$. Clearly $S \simeq T$ in $\mathscr{C} / \mathscr{S}$. Let $\bar{f}: G / S \rightarrow H / T$ and $\bar{g}: H / T \rightarrow G / S$ be the homomorphisms induced be $f$ and $g$, respectively. Then $\bar{g} \bar{f}=k$ and $\bar{f} \bar{g}=k$. Hence $G / S \simeq H / T$ in $\mathscr{A} / \mathscr{B}$.

Let $G_{t}$ be the torsion subgroup of $G$. If $G \simeq H$ in $\mathscr{A} / \mathscr{B}$, then $G_{t} \simeq H_{t}$ in $\mathscr{N} / \mathscr{S}$ and $G / G_{t} \dot{\sim} H / H_{t}$. Moreover, if $G$ and $H$ split, $G_{t} \simeq H_{t}$ in $\mathscr{A} / \mathscr{S}$, and $G / G_{t} \dot{\simeq} H / H_{t}$, then $G \simeq H$ in $\mathscr{A} / \mathscr{S}$. We now reduce the study of isomorphism in $\mathscr{C} / \mathscr{B}$ from torsion groups to primary groups.

Proposition 3.3. Let $G$ and $H$ be torsion groups, and let $G_{p}$ and $H_{p}$ be the $p$ components of $G$ and $H$, respectively. Then $G \simeq H$ in $\mathscr{A} \mid \mathscr{S}$ if and only if $G_{p} \simeq H_{p}$ in $\mathscr{A} / \mathscr{B}$ for all primes $p$ and $G_{p} \simeq H_{p}$ for almost all primes $p$.

Proof. Suppose $G \simeq H$ in $\mathscr{A} / \mathscr{B}$. Then there exists an integer $k>0$ and homomorphisms $f: G \rightarrow H$ and $g: H \rightarrow G$ such that $g f=k$ $f g=k$. Since $f\left(G_{p}\right) \subseteq H_{p}$ and $g\left(H_{p}\right) \subseteq G_{p}, G_{p} \simeq H_{p}$ in $\mathscr{A} / \mathscr{B}$ for each prime $p$. Let $p$ be a prime which does not divide $k$. Let $f_{p}=$ $f \mid G_{p}$. Suppose $f_{p}(x)=0$ for some $x \in G_{p}$. Let $o(x)=p^{m}$ and write 
$1=a k+b p^{m}$. Now $x=a k x=a g f_{p}(x)=0$. Thus $f_{p}$ is a monomorphism. Let $y \in H_{p}, o(y)=p^{n}$. Write $1=a^{\prime} k+b^{\prime} p^{n}$. Now $f_{p} g\left(a^{\prime} y\right)=$ $a^{\prime} f_{p} g(y)=a^{\prime} k y=y$. Thus $f_{p}$ is an epimorphism. Hence $G_{p} \simeq H_{p}$ for each prime $p$ not dividing $k$.

The converse is clear.

Let $G$ be a divisible $p$-group and $A=\Sigma\left\langle a_{\alpha}\right\rangle$ a bounded subgroup of $G$. Embed each $\left\langle a_{\alpha}\right\rangle$ in a summand $G_{\alpha} \simeq Z\left(p^{\infty}\right)$ of $G$. Then $G_{\alpha} \mid\left\langle a_{\alpha}\right\rangle \simeq G_{\alpha}$. Thus $G / A \simeq G$. Given two divisible groups $D$ and $E$, it now follows from 3.1 that $D \simeq E$ in $\mathscr{A} / \mathscr{B}$ implies that $D \simeq E$. An application of 3.2 yields the next proposition.

Proposition 3.4. Let $G=D \oplus R$ and $H=D^{\prime} \oplus R^{\prime}$ where $D$ and $D^{\prime}$ are divisible and $R$ and $R^{\prime}$ are reduced. Then $G \simeq H$ in $\mathscr{A} / \mathscr{S}^{\prime}$ if and only if $D \simeq D^{\prime}$ in $\mathscr{A}$ and $R \simeq R^{\prime}$ in $\mathscr{A} / \mathscr{S}$.

Let $G$ be a group. Then $G^{1}=\bigcap_{0<n>\omega} n G$ is a subgroup of $G$ called the elements of infinite height of $G$. If $G$ is a p-group, then $G^{1}=\bigcap_{0<n<\omega} p^{n} G$.

To see that the property of being a direct sum of cyclic groups is not preserved under isomorphism in $\mathscr{A} / \mathscr{B}$, we need only consider the Prufer group, $P$, which is generated by elements $a_{1}, a_{2}, \cdots, a_{n}, \cdots$ with the relations

$$
p a_{1}=0, a_{1}=p a_{2}=p^{2} a_{3}=\cdots=p^{n} a_{n+1}=\cdots .
$$

Now $B=\sum_{n \geqq 2}\left\langle a_{n}-p a_{n-1}\right\rangle$ is a basic subgroup of $P, P^{1}=\left\langle a_{1}\right\rangle \simeq C(p)$, and $P / P^{1} \simeq B$. (See [4]). So $P \simeq B$ in $\mathscr{C} / \mathscr{S}$. We shall point out some other properties which are related to direct sums of cyclic groups and direct sums of countable groups and which are not preserved by isomorphisms in $\mathscr{O} / \int_{\text {. }}$. First a further look at direct sums of cyclic groups.

Proposition 3.5. The following are equivalent for a $p$-group $G$.

(i) $G$ is isomorphic in $\mathscr{C} / \mathscr{B}$ to a direct sum of cyclic groups.

(ii) $G / A$ is a direct sum of cyclic groups for some bounded subgroup $A \subseteq G$.

(iii) There is a direct sum of cyclic groups $C$ and a bounded subgroup $B \cong C$ such that $G \simeq C / B$.

(iv) Every subgroup of $G$ is isomorphic in $\mathscr{A} / \mathscr{B}$ to a direct sum of cyclic groups.

(v) $\operatorname{Ext}(G, H)^{1}$ is bounded for all p-groups $H$.

Proof. It is clear that (ii), (iii), and (iv) each imply (i) and that (iii) implies (iv). That (i) implies (ii) follows from 3.1., and (ii) if and only if $(\mathrm{v})$ is Theorem $27^{\prime}$ of [10]. 
Suppose $G \simeq H$ in $\mathscr{A} / \mathscr{B}, H$ a direct sum of cyclic groups. Then there exists a bounded subgroup $B \cong H$, an integer $n \geqq 0$, and a monomorphism $f: p^{n} G \rightarrow H / B$ with bounded cokernel. So $p^{n} G \simeq T / B$ where $p^{k} H \subseteq T \subseteq H$ for some $k \geqq 0$. By 50.1 in [4], there exists a group $T \leqq C$ such that the isomorphism $p^{n} G \simeq T / B$ can be extended to an isomorphism $G \simeq C / B$. Thus $p^{n} G \subseteq T \leqq H$ and $C$ is a direct sum of cyclic groups. Hence (i) implies (iii).

Proposition 3.6. Let $G$ be a $p$-group. If $G$ is isomorphic in $\mathscr{A} / \mathscr{B}$ to a direct sum of cyclic groups, then $G^{1}$ is bounded. If $G$ is a direct sum of countable groups, the converse holds.

Proof. The first statement follows from (ii) of 3.5. If $G$ is a direct sum of countable groups and $p^{k} G^{1}=0$, then

$$
p^{k} G \simeq\left(G / G^{1}\right) /\left(G\left[p^{k}\right] / G^{1}\right)
$$

and $G / G^{1}$ is a direct sum of cyclic groups. The result follows from (iii) of 3.5.

Let $G$ be a group. If every infinite subgroup of $G$ can be embedded in a summand of the same cardinality, then $G$ is Fuchs five [9]. It is easy to see that if $G$ is a direct sum of countable groups, then $G$ is Fuchs five. If $G$ has no elements of infinite height and if for every infinite subgroup $H$ of $G,\left|(G / H)^{1}\right| \leqq|H|$, then $G$ is a $Q$-group [9]. If whenever $G / H$ is divisible, $H$ a subgroup of $G$, we have $|H|=|G|$, then $G$ is starred [9]. Finally, $G$ is fully starred if every subgroup of $G$ is starred. F. Richman and J. Irwin [9] showed that if

$$
0 \longrightarrow G \longrightarrow H \longrightarrow K \longrightarrow 0
$$

is exact and if $G$ and $K$ are starred, then $H$ is starred. Also in [9], it is shown that if $G$ is Fuchs five and $G^{1}=0$, then $G$ is a $Q$-group, and if $G$ is a $Q$-group, then $G$ is fully starred.

Proposition 3.7. Let $G \simeq H$ in $\mathscr{A} / \mathscr{S}$. Then $G$ is fully starred if and only if $H$ is fully starred.

Proof. Suppose $H$ is fully starred. Let $A$ be a bounded subgroup of $G$ such that $n H \simeq S / A$ where $m G \leqq S \leqq G, m$ and $n$ positive integers. Then $S / A$ is fully starred and $A$, being a direct sum of cyclic groups, is starred. Since

$$
0 \longrightarrow A \longrightarrow S \longrightarrow S / A \longrightarrow 0
$$

is exact, it follows that $S$ is starred. Now 


$$
0 \longrightarrow S \longrightarrow G \longrightarrow G / S \longrightarrow 0
$$

is exact with $G / S$ bounded. Thus $G$ is starred. Let $K$ be a subgroup of $G$. Then $(m K+A) / A$ is a subgroup of $S / A$ and so $(m K+A) / A \simeq$ $m K / m K \cap A$ is starred. By the exactness of

$$
0 \longrightarrow m K \cap A \longrightarrow m K \longrightarrow m K / m K \cap A \longrightarrow 0
$$

and

$$
0 \longrightarrow K[m] \longrightarrow K \longrightarrow m K \longrightarrow 0
$$

it now follows that $K$ is starred. Hence $G$ is fully starred.

The following example shows that a $p$-group can have no elements of infinite height, be isomorphic in $\mathscr{A} / \mathscr{B}$ to a direct sum of cyclic groups, and not be a $Q$-group.

Example 3.8. Let $B \simeq C(p) \oplus C\left(p^{2}\right) \oplus \cdots$ where $C\left(p^{n}\right)$ is cyclic of order $p^{n}$. Then $\bar{B}$ is uncountable, has no elements of infinite height and is not fully starred. Let $C$ be countable with $C^{1} \neq 0$. In [9] it is shown that $\operatorname{Tor}(\bar{B}, C)$ is fully starred, has no elements of infinite height, and is not a $Q$-group. The exact sequence

$$
0 \longrightarrow C(p) \longrightarrow P \longrightarrow B \longrightarrow 0
$$

with $P$ the Prufer group and $C(p)=P^{1}$ yields the exact sequence

$$
0 \longrightarrow \operatorname{Tor}(\bar{B}, C(p)) \longrightarrow \operatorname{Tor}(\bar{B}, P) \longrightarrow \operatorname{Tor}(\bar{B}, B) \text {. }
$$

But Tor $(\bar{B}, C(p))$ is $p$-bounded and Tor $(\bar{B}, B)$ is a direct sum of cyclic groups. Thus Tor $(\bar{B}, P)$ is the desired group.

4. U1m's theorem in $\mathscr{A} / \mathscr{B}$. All groups considered in this section are reduced primary Abelian groups for a fixed prime $p$. Let $G$ be such a group and let $\alpha$ be an ordinal number. We define $p^{\alpha} G$ inductively as follows: $p^{0} G=G, p^{\alpha} G=p\left(p^{\alpha-1} G\right)$ if $\alpha-1$ exists, and $p^{\alpha} G=\bigcap_{\beta<\alpha} p^{\beta} G$ if $\alpha$ is a limit ordinal. The dimension $f_{G}(\alpha)$ of the vector space $\left(p^{\alpha} G\right)[p] /\left(p^{\alpha+1} G\right)[p]$ is called the $\alpha$ th Ulm invariant of $G$. If $G$ is a direct sum of cyclic groups, then $f_{G}(n)$ is just the number of cyclic summands of $G$ of order $p^{n+1}$. We define $G$ inductively as follows: $G^{0}=G, G^{\alpha}=p^{\omega}\left(G^{\alpha-1}\right)$ if $\alpha-1$ exists, and $G^{\alpha}=\bigcap_{\beta<\alpha} G^{\beta}$ if $\alpha$ is a limit ordinal. Since $G$ is reduced, there is a smallest ordinal $\tau,|\tau| \leqq|G|$, such that $G^{\tau}=0 . \quad \tau$ is called the length of $G$. The quotient groups $G^{\alpha} / G^{\alpha+1}, \alpha<\tau$, are called the Ulm factors of $G$. All the Ulm factors except possibly the last, if it exists, are unbounded. Moreover, if $G$ is a direct sum of countable groups, then $G^{\alpha} / G^{\alpha+1}$ is a direct sum of cyclic groups for each $\alpha<\tau$. The relationship between 
$p^{\alpha} G$ and $G^{\alpha}$ is given by $G^{\alpha}=p^{\omega \alpha} G$. Finally an element $x \in G$ is said to have height $\alpha$ in $G$ if $x \in p^{\alpha} G, x \notin p^{\alpha+1} G$, and we write $H_{G}(x)=\alpha$.

One of the most important theorems in the theory of Abelian groups is Ulm's theorem, which states that two countable reduced $p$ groups are isomorphic if and only if their corresponding Ulm factors are isomorphic, or equivalently, if and only if they have the same Ulm invariants. This result has been extended to direct sums of countable reduced $p$-groups by G. Kolettis [12]. Another proof is given by $\mathrm{P}$. Hill [7].

The main result in this section is an "Ulm's theorem" for direct sums of countable reduced $p$-groups in the category $\mathscr{A} / \mathscr{B}$. In the course of the proof of this theorem, several subsidiary results are obtained. These will be stated as they arise.

Let $G$ and $H$ be reduced $p$-groups. Suppose

(I) there is an integer $k \geqq 0$ such that for all integers $n \geqq 0$ and $r \geqq 0$

$$
\sum_{j=0}^{r} f_{G}(n+k+j) \leqq \sum_{j=0}^{r+2 k} f_{H}(n+j), \sum_{j=0}^{r} f_{H}(n+k+j) \leqq \sum_{j=0}^{r+2 k} f_{G}(n+j),
$$

and

(II) $f_{G}(\alpha)=f_{I I}(\alpha)$ for all $\alpha \geqq \omega$.

In [1] Beaumont and Pierce show that (I) and (II) are necessary and sufficient for two countable reduced $p$-groups to be quasi-isomorphic. In [2] Beaumont and Pierce show that (I) is necessary and sufficient for two direct sums of cyclic $p$-groups to be quasi-isomorphic. In view of Kolettis extension of Ulm's theorem, it is natural to ask if (I) and (II) are necessary and sufficient for two direct sums of countable reduced $p$-groups to be quasi-isomorphic. The answer is in the affirmative, and the proof is straightforward since $n G \subseteq S \subseteq G$ and $G$ a direct sum of countable groups implies that $S$ is a direct sum of countable groups. (For a proof of this latter fact, see Irwin and Richman [9].) In [8] P. Hill proves the following more general statement. If $G$ and $H$ are reduced $p$-groups with $G / G^{1}$ and $H / H^{1}$ direct sums of cyclic groups, then (I) and (II) are necessary and sufficient for $G$ and $H$ to be quasi-isomorphic. Hill also includes in [8] a short and informative proof of the previously mentioned result by Beaumont and Pierce [2].

While the following lemma does not appear in [8], it is an immediate consequence of a result in [8]. Moreover, it has an application later on.

Lemma 4.1. Let $G$ and $H$ be direct sums of cyclic p-groups which satisfy (I). Suppose $f_{G}(0)=|G||H| \boldsymbol{\aleph}_{0}=f_{H}(0)$. Then

$$
G=G_{0} \oplus G_{1} \oplus \cdots \oplus G_{2 k} \quad \text { and } \quad H=H_{0} \oplus H_{1} \oplus \cdots \oplus H_{2 k}
$$


where

$$
p^{k} G_{0} \simeq H_{0}, p^{k-1} G_{1} \simeq H_{1}, \cdots, G_{k} \simeq H_{k}, G_{k+1} \simeq p H_{k+1}, \cdots, G_{2 k} \simeq p^{k} H_{2 k} .
$$

Proof. For each integer $n \geqq 0$, let $A_{n}$ be the initial segment of ordinals less than the first ordinal of cardinality $f_{G}(n)$. Similarly, let $B_{n}$ be the initial segment of ordinals less than the first ordinal of cardinality $f_{I I}(n)$. Define

$$
\begin{aligned}
& A=\left\{(n, \alpha) \mid 0 \leqq n<\omega, \alpha \in A_{n}\right\}, \\
& B=\left\{(n, \beta) \mid 0 \leqq n<\omega, \beta \in B_{n}\right\} .
\end{aligned}
$$

Write $G=\sum_{n<\omega} G_{n}$ where $G_{n}$ is a direct sum of cyclic groups of order $p^{n+1}$. Then $G_{n}=\sum_{\alpha \epsilon_{n}}\left\langle a_{n, \alpha}\right\rangle$ and $(n, \alpha) \rightarrow a_{n, \alpha}$ is an equivalence between $A$ and a basis of $G$. Write $H=\sum_{n<\omega} \sum_{\beta \epsilon_{n}}\left\langle b_{n, \beta}\right\rangle$ where $\left\langle b_{n, \beta}\right\rangle \simeq C\left(p^{n+1}\right)$ for each $\beta \in B_{n}$. Then $b_{n, \beta} \rightarrow(n, \beta)$ is an equivalence between $B$ and a basis of $H$. In [8] Hill shows that there is an equivalence between $B$ and $A$ which alters indicies by no more than $k$, the index of the element $(n, \gamma)$ of $A$ or $B$ being $n$. Thus we have an equivalence $\pi$ between a basis of $H$ and a basis of $G$ which alters exponents by no more than $k$. For $0 \leqq i \leqq 2 k$, define

$$
\begin{aligned}
& G_{i}=\sum\left\{\left\langle a_{n, \alpha}\right\rangle \mid \pi\left(b_{m, \beta}\right)=a_{n, \alpha}, n-m=k-i\right\}, \\
& H_{i}=\sum\left\{\left\langle b_{m, \beta}\right\rangle \mid \pi\left(b_{m, \beta}\right)=a_{n, \alpha}, n-m=k-i\right\} .
\end{aligned}
$$

The lemma follows.

Let $G$ be a group. A subgroup $H$ of $G$ is essential in $G$ if $K \subseteq G$ and $K \cap H=0$ imply $K=0$. A group $X$ is an $n$-extension of $G$ if $G$ is essential in $X$ and $n X=G$. Every group has an $n$ extension. (See Walker [15].) In fact $G^{n}$ is an $n$-extension of $G$ if $G^{n} / G=(D / G)[n]$ where $D$ is a divisible hull of $G$. This notion is used in the proof of the following corollary.

CoROLlary 4.2. Let $G$ and $H$ be direct sums of cyclic p-groups which satisfy (I). Then there are subgroups $S \subseteq G$ and $T \subseteq H$ such that $p^{k} G \subseteq S, p^{k} H \subseteq T$, and $S \simeq T$.

Proof. If $k=0$, then $f_{G}(n)=f_{H}(n)$ for all integers $n \geqq 0$ and so $G \simeq H$. Assume $k>0$ and let $G^{*}$ and $H^{*}$ be such that $p G^{*}=G$, $p H^{*}=H$, and $f_{G^{*}}(0)=\left|G^{*}\right|\left|H^{*}\right| \boldsymbol{\aleph}_{0}=f_{I I^{*}}(0)$. For example, take $p$-extensions of $G$ and $H$, respectively, and then add on summands $\sum_{\boldsymbol{*}} C(p)$ where $\boldsymbol{\aleph}=\left|G^{*}\right|\left|H^{*}\right| \boldsymbol{\aleph}_{0}$. Write $k=m+1$. Then

$$
\begin{aligned}
\sum_{j=0}^{r} f_{G^{*}}(n+k+j) & =\sum_{j=0}^{r} f_{p G^{*}}(n+m+j) \leqq \sum_{j=0}^{r+2 m} f_{p H^{*}}(n+j) \\
& =\sum_{j=0}^{r+2 m} f_{H^{*}}(n+1+j) \leqq \sum_{j=0}^{r+2 k} f_{H^{*}}(n+j) .
\end{aligned}
$$


for all integers $n \geqq 0$ and $r \geqq 0$. Similarly

$$
\sum_{j=0}^{r} f_{H^{*}}(n+k+j) \leqq \sum_{j=0}^{r+2 k} f_{G^{*}}(n+j) .
$$

Now write $G^{*}=G_{0} \oplus \cdots \oplus G_{2 k}$ and $H^{*}=H_{0} \oplus \cdots \oplus H_{2 k}$ according to 4.1. Let $S^{*}=p^{k} G_{0} \oplus p^{k-1} G_{1} \oplus \cdots \oplus G_{k} \oplus \cdots \oplus G_{2 k}$ and let $T^{*}=$ $H_{0} \oplus \cdots \oplus H_{k} \oplus p H_{k+1} \oplus \cdots \oplus p^{k} H_{2 k}$. Then $p^{k} G^{*} \subseteq S^{*}, p^{k} H^{*} \subseteq T^{*}$, and $S^{*} \simeq T^{*}$. Now $p^{k} G=p^{k+1} G^{*} \subseteq p S^{*} \subseteq p G^{*}=G, p^{k} H \subseteq p T^{*} \subseteq H$, and $p S^{*} \simeq p T^{*}$.

Lemma 4.3. Let $H$ be a reduced p-group and $n$ and $r$ positive integers. Then

$$
\operatorname{dim}\left(\left(p^{n} H\right)[p] /\left(p^{n+r+1} H\right)[p]\right)=\sum_{j=0}^{r} f_{H}(n+j) .
$$

\section{Proof. Since}

$$
\left(p^{n} H\right)[p] /\left(p^{n+2} H\right)[p] \simeq\left(\left(p^{n+1} H\right)[p] /\left(p^{n+2} H\right)[p]\right) \oplus X
$$

where $X \simeq\left(p^{n} H\right)[p] /\left(p^{n+1} H\right)[p]$, the lemma follows from induction and the definition of $f_{H}$.

LEMMA 4.4. Let $G$ be a reduced p-group, $k \geqq 0$, and $A \subseteq G\left[p^{k}\right]$. Then for all integers $n \geqq 0$ and $r \geqq 0$,

$$
\sum_{j=0}^{r} f_{G / A}(n+j) \leqq \sum_{j=0}^{r+k} f_{G}(n+j) \quad \text { and } \quad \sum_{j=0}^{r} f_{G}(n+k+j) \leqq \sum_{j=0}^{r+k} f_{G / A}(n+j) .
$$

Proof. Let $B$ be a basic subgroup of $G$, and write $B=\sum_{i=1} B_{i}$ with each $B_{i}$ a direct sum of cyclic groups of order $p^{i}$. Let

$$
\left\{x_{i}+A+\left(\left(p^{n+r+1} G+A\right) / A\right)[p]\right\}_{i \in I}
$$

be a basis of $\left(\left(p^{n} G+A\right) / A\right)[p] /\left(\left(p^{n+r+1} G+A\right) / A\right)[p]$, with each $x_{i} \in p^{n} G$, $x_{i} \notin p^{n+r+1} G$. Applying 4.3 to the group $G / A$, we have

$$
|I|=\sum_{j=0}^{r} f_{G / A}(n+j) \text {. }
$$

Since $n \leqq H_{G}\left(x_{i}\right)<n+r+1$ and $E\left(x_{i}\right) \leqq k+1, x_{i}=c_{i}+d_{i}$ where $0 \neq c_{i} \in B_{n+1} \oplus \cdots \oplus B_{n+k+r+1}, c_{i} \notin p^{n+r+1} G$, and $d_{i} \in p^{n+r+1} G$. Let $C=$ $B_{n+1} \oplus \cdots \oplus B_{n+k+r+1}$. Now

$$
\frac{\left(\left(C+p^{n+r+1} G+A\right) / A\right)[p]}{\left(\left(p^{n+r+1} G+A\right) / A\right)[p]} \supseteqq \frac{\left(\left(p^{n} G+A\right) / A\right)[p]}{\left(\left(p^{n+r+1} G+A\right) / A\right)[p]} .
$$

Hence 


$$
\begin{aligned}
\sum_{j=0}^{r} f_{G \mid A}( & n+j)=|I| \\
& \leqq \operatorname{dim}\left(\left(\left(C+p^{n+r+1} G+A\right) / A\right)[p] /\left(\left(p^{n+r+1} G+A\right) / A\right)[p]\right) \\
& \leqq \operatorname{dim}\left(\left(\left(\left(C+p^{n+r+1} G+A\right) / A\right) /\left(\left(p^{n+r+1} G+A\right) / A\right)\right)[p]\right) \\
& \left.=\operatorname{dim}\left(\left(\left(C+p^{n+r+1} G+A\right) / p^{n+r+1} G+A\right)\right)[p]\right) \\
& =\operatorname{dim}\left(\left(C /\left(C\left(p^{n+r+1} G+A\right)\right)\right)[p]\right) \\
& \leqq \operatorname{dim}(C[p])=\sum_{j=0}^{r+k} f_{B}(n+j)=\sum_{j=0}^{r+k} f_{G}(n+j) .
\end{aligned}
$$

Since $f_{p^{k} G}(m)=f_{G}(m+k)$ for all $m \geqq 0$, and $G\left[p^{k}\right] / A \subseteq(G / A)\left[p^{k}\right]$, applying the first inequality to $G / A$ and the subgroup $G\left[p^{k}\right] / A$ yields

$$
\begin{aligned}
\sum_{j=0}^{r} f_{G}(n+k+j) & =\sum_{j=0}^{r} f_{p^{k} G}(n+j) \\
& =\sum_{j=0}^{r} f_{(G / A) /\left(G\left[p^{k}\right] / A\right)}(n+j) \leqq \sum_{j=0}^{r+k} f_{G / A}(n+j) .
\end{aligned}
$$

This completes the proof.

CoROllaRY 4.5. Let $G$ and $H$ be reduced p-groups with $G \simeq H$ in $\mathscr{A} / \mathscr{B}$. Then $G$ and $H$ satisfy (I).

Proof. By 3.1., there is a $k \geqq 0$ and a homomorphism $f: G \rightarrow H$ such that $\operatorname{Ker} f$ and Coker $f$ are bounded by $p^{k}$. Let $A=\operatorname{Ker} f$ and $T=\operatorname{Im} f$. By 4.4,

$$
\sum_{j=0}^{r} f_{G / A}(n+j) \leqq \sum_{j=0}^{r+k} f_{G}(n+j) \text { and } \sum_{j=0}^{r} f_{G}(n+k+j) \leqq \sum_{j=0}^{r+k} f_{G / A}(n+j)
$$

for all $n \geqq 0$ and all $r \geqq 0$. Now

$$
p^{k} H \subseteq T \leqq H,\left(p^{n+k} H\right)[p] /\left(p^{n+r+k+1} H\right)[p] \subseteq\left(p^{n} T\right)[p] /\left(p^{n+r+k+1} H\right)[p]
$$

and the latter is an image of $\left(p^{n} T\right)[p] /\left(p^{n+r+k+1} T\right)[p]$. Also

$$
\left(p^{n} T\right)[p] /\left(p^{n+r+k+1} T\right)[p]\left(p^{n} H\right)[p] /\left(p^{n+r+k+1} T\right)[p]
$$

and the latter is an image of $\left(p^{n} H\right)[p] /\left(p^{n+r+2 k+1} H\right)[p]$. Thus, applying 4.3 and 4.4 ,

$$
\sum_{j=0}^{r} f_{H}(n+k+j) \leqq \sum_{j=0}^{r+k} f_{T}(n+j)=\sum_{j=0}^{r+k} f_{G / A}(n+j) \leqq \sum_{j=0}^{r+2 k} f_{G}(n+j)
$$

and

$$
\sum_{j=0}^{r} f_{G}(n+k+j) \leqq \sum_{j=0}^{r+k} f_{G / A}(n+j)=\sum_{j=0}^{r+k} f_{T}(n+j) \leqq \sum_{j=0}^{r+2 k} f_{H}(n+j) .
$$

CoRollary 4.6. Let $G$ and $H$ be reduced p-groups with basic 
subgroups $B$ and $C$, respectively. Then $B \dot{\simeq} C$ whenever $G \simeq H$ in $\mathscr{A} / \mathscr{B}$.

Proof. $B$ and $C$ satisfy (I) whenever $G$ and $H$ do. Now apply the result in [2].

CoROllary 4.7. Let $\bar{B}$ and $\bar{C}$ be closed p-groups with basic subgroups $B$ and $C$, respectively. Then $\bar{B} \simeq \bar{C}$ in $\mathscr{A} / \mathscr{B}$ if and only if $\bar{B} \dot{\sim} \bar{C}$.

Proof. $\bar{B} \dot{\simeq} \bar{C}$ in $\mathscr{A} / \mathscr{B}$ implies $B \dot{\simeq} C$ by 4.6 , and $B \dot{\simeq} C$ implies $\bar{B} \simeq \bar{C}$ is Theorem 3.11 in [3].

CoRollary 4.8. Let $G$ and $H$ be reduced p-groups and suppose $G / G^{1}$ and $H / H^{1}$ are direct sums of cyclic groups. If $G \simeq H$ in $\mathscr{A} \mid \mathscr{S}$ and $G^{1} \simeq H^{1}$, then $G \dot{\simeq} H$.

Proof. In [8], Hill shows that $G \dot{\simeq} H$ if $G$ and $H$ satisfy (I) and (II) and $G / G^{1}$ and $H / H^{1}$ are direct sums of cyclic groups. The corollary now follows from 4.5 .

In view of 4.6 and 4.7 , it is natural to ask the following question. If $G \simeq H$ in $\mathscr{A} / \mathscr{B}$, is $G \dot{\sim} H$ whenever $G$ and $H$ are reduced $p$ groups without elements of infinite height? The answer is no and 3.8 furnishes us with a counterexample. Specifically, there is a $p$ group $G$ and a subgroup $A \cong G[p]$ such that

(i) $G^{1}=0$,

(ii) $G$ is not a direct sum of cyclic groups,

(iii) $(G / A)^{1}=0$,

(iv) $G / A$ is a direct sum of cyclic groups. Now $G \simeq G / A$ in $\mathscr{A} / \mathscr{B}$, but $G \dot{\simeq} G / A$ contradicts (ii) and (iv).

LemMa 4.9. Let $G$ and $H$ be direct sums of cyclic p-groups such that $f_{G}(0)=|G||H| \boldsymbol{\aleph}_{0}=f_{H}(0)$. Suppose there is an integer $k \geqq 0$ such that for all integers $n \geqq 0$ and $r \geqq 0$

$$
\sum_{j=0}^{r} f_{H}(n+j) \leqq \sum_{j=0}^{r+k} f_{G}(n+j) \quad \text { and } \quad \sum_{j=0}^{r} f_{G}(n+k+j) \leqq \sum_{j=0}^{r+k} f_{H}(n+j) .
$$

Then there is an equivalence of a basis of $G$ onto a basis of $H$ such that exponents are never increased and are not decreased by more than $k$.

Proof. For each integer $n \geqq 0$, define $A_{n}$ and $B_{n}$ as in the proof of 4.1. Also define $A$ and $B$ as in 4.1. Call the first component of 
the element $(n, \gamma)$ of $A$ or $B$ the index of the element. It was pointed out in the proof of 4.1 that $A$ is in one-to-one correspondence with a basis of $G$ such that if the index of an element of $A$ is $n$, then the exponent of the corresponding basis element of $G$ is $n+1$. There is a similar one-to-one correspondence between $B$ and a basis of $H$. Hill [8] has shown that (I) implies the existence of an equivalence between $A$ and $B$ which alters indicies by no more than $k$. By a close examination of Hill's proof, it is seen that the stronger inequalities in the hypothesis of 4.9 imply the existence of an equivalence $\theta$ from $A$ onto $B$ with the index of $\theta(n, \alpha)$ between $n-k$ and $n$. Thus there is an equivalence from a basis of $G$ onto a basis of $H$ such that exponents are never increased and are not decreased by more than $k$.

Corollary 4.10. Let $G$ and $H$ be direct sums of cyclic p-groups and $k$ a positive integer such that for all integers $n \geqq 0$ and $r \geqq 0$

$$
\sum_{j=0}^{r} f_{I I}(n+j) \leqq \sum_{j=0}^{r+k} f_{G}(n+j) \quad \text { and } \quad \sum_{j=0}^{r} f_{G}(n+k+j) \leqq \sum_{j=0}^{r+k} f_{H I}(n+j) \text {. }
$$

Then there is a subgroup $S \subseteq G$ such that $p^{k} G \cong S$ and $S \simeq H$.

Proof. If $k=0$, then $f_{G}(n)=f_{H}(n)$ for all $n \geqq 0$ and so $G \simeq H$. Assume $k \geqq 1$, let $p G^{*}=G, p H^{*}=H$, and $f_{G^{*}}(0)=\left|G^{*} \| H^{*}\right| \boldsymbol{\aleph}_{0}=$ $f_{H^{*}}(0)$. Write $k=m+1$. Let $n \geqq 0$ and $r \geqq 0$. Then

$\sum_{j=0}^{r} f_{G^{*}}(n+k+j)=\sum_{j=0}^{r} f_{p G^{*}}(n+m+j) \leqq \sum_{j=0}^{r+m} f_{p I I^{*}}(n+j) \leqq \sum_{j=0}^{r+k} f_{I I^{*}}(n+j)$.

If $n=0$, then

$$
\sum_{j=0}^{r} f_{H^{*}}(j)=f_{H^{*}}(0)=f_{G^{*}}(0)=\sum_{j=0}^{r+k} f_{G^{*}}(j)
$$

If $n \geqq 1$, say $n=m+1$, then

$$
\sum_{j=0}^{r} f_{H H^{*}}(n+j)=\sum_{j=0}^{r} f_{p H^{*}}(m+j) \leqq \sum_{j=0}^{r+k} f_{p G^{*}}(m+j)=\sum_{j=0}^{r+k} f_{G^{*}}(n+j) .
$$

Thus $G^{*}$ and $H^{*}$ satisfy the hypothesis of 4.9 . Let $A$ be a basis of $G^{*}$ and $B$ a basis of $H^{*}$. Let $\pi$ be an equivalence of $A$ onto $B$ determined by 4.9 . For $0 \leqq i \leqq k$, define

$$
\begin{aligned}
& G_{i}=\sum\{\langle a\rangle \mid a \in A, \pi(a)=b, E(a)-E(b)=k-i\}, \\
& H_{i}=\sum\{\langle b\rangle \mid b \in B, \pi(a)=b, E(a)-E(b)=k-i\} .
\end{aligned}
$$

Clearly $\quad G^{*}=G_{0} \oplus \cdots \oplus G_{k}, H^{*}=H_{0} \oplus \cdots \oplus H_{k}, p^{k-i} G_{i} \simeq H_{i} \quad$ for $i=1, \cdots, k$. Let $S^{*}=p^{k} G_{0} \oplus p^{k-1} G_{1} \oplus \cdots \oplus G_{k}$. Then $S^{*} \simeq H^{*}$ 
and $p^{k} G^{*} \subseteq S^{*} \subseteq G$. Let $S=p S^{*}$. Now $S \simeq H$ and $p^{k} G=p^{k+1} G^{*} \subseteq$ $p S^{*} \leqq p G^{*}=G$. This completes the proof.

CoRollary 4.11. Let $G$ be a direct sum of cyclic p-groups, $k \geqq 0$, and $A \subseteq G\left[p^{k}\right]$. Suppose $G / A$ is a direct sum of cyclic groups. Then there is a subgroup $S \subseteq G$ such that $p^{k} G \subseteq S$ and $G / A \simeq S$.

Proof. Apply 4.4 to see that $G$ and $G / A$ satisfy the hypothesis of 4.10 with $H=G / A$.

Corollary 4.11 is a generalization of the well-known fact that the quotient of a finite group $G$ is isomorphic to a subgroup of $G$.

Proposition 4.12. Let $G$ and $H$ be reduced $p$-groups with $G \simeq H$ in $\mathscr{A} / \mathscr{B}$. Then $p^{\alpha} G \simeq p^{\alpha} H$ in $\mathscr{A} / \mathscr{B}$, and $G^{\alpha} / G^{\alpha+1} \simeq H^{\alpha} / H^{\alpha+1}$ in $\mathscr{A} 1 . \mathscr{S}$.

Proof. This is a corollary of 3.2 .

THEOREM 4.13. Let $G$ and $H$ be reduced p-groups with $G \simeq H$ in $\mathscr{A} / \mathscr{B}$. Then there exists an integer $k \geqq 0$ such that for all ordinals $\alpha$ and for all $r \geqq 0$

$$
\sum_{j=0}^{r} f_{G}(\alpha+k+j) \leqq \sum_{j=0}^{r+2 k} f_{H}(\alpha+j)
$$

and

$$
\sum_{\jmath=0}^{r} f_{H}(\alpha+k+j) \leqq \sum_{j=0}^{r+2 k} f_{G}(\alpha+j)
$$

Proof. Since $p^{\alpha} G \simeq p^{\alpha} H$ in $\mathscr{A} / \mathscr{B}, p^{\alpha} G$ and $p^{\alpha} H$ satisfy (I). Moreover, by 3.1 and $4.5, k$ is the same for each $\alpha$. Since $f_{G}(\alpha+n)=f_{p^{\alpha} G}(n)$, the theorem follows.

Let $G$ and $H$ be countable reduced $p$-groups and suppose $G \simeq H$ in $\mathscr{A} / \mathscr{B}$. By 4.12 and 4.6 , we see that the corresponding Ulm factors of $G$ and $H$ are quasi-isomorphic. The following example shows that the converse does not hold.

EXAMPLE 4.14. Let $G$ and $H$ be countable groups whose respective Ulm factors are

$$
G_{n} \simeq C\left(p^{n+1}\right) \oplus C\left(p^{n+2}\right) \oplus \cdots \quad \text { for } \quad n \geqq 0, G_{\omega}=0
$$

and

$$
H_{n} \simeq C\left(p^{2 n+2}\right) \oplus C\left(p^{2 n+3}\right) \oplus \cdots \quad \text { for } \quad n \geqq 0, H_{\omega}=0
$$


Clearly $G_{n} \dot{\sim} H_{n}$. In fact $p^{n+1} H_{n} \simeq G_{n}$. Suppose $G \simeq H$ in $\mathscr{A} / \mathscr{B}$. By 3.1, there is an integer $n \geqq 0$ and homomorphisms $f: G \rightarrow H$ and $g: H \rightarrow G$ such that $g f=p^{n}$ and $f g=p^{n}$. Now $f$ and $g$ induce homomorphisms $f: G_{n} \rightarrow H_{n}$ and $g: H_{n} \rightarrow G_{n}$ such that $g f=p^{n}$ and $f g=p^{n}$. Let $x$ generate a summand of $G_{n}$ of order $p^{n+1}$. Then $f(x)=p^{n+1} y$ for some $y \in H_{n}$. Thus $0 \neq p^{n} x=g f(x)=p^{n+1} g(y)$ which is a contradiction. Hence $G$ and $H$ are not isomorphic in $\mathscr{A} / \mathscr{S}$.

In view of Example 4.14, we make the following definition.

DeFinition 4.15. Let $\left\{G_{\alpha}\right\}_{\alpha \in I}$ and $\left\{H_{\alpha}\right\}_{\alpha \in I}$ be families of groups over the same index set. These families are uniformly quasi-isomorphic if there is an integer $k \geqq 0$ and for each $\alpha \in I$, subgroups $S_{\alpha} \subseteq G_{\alpha}$ and $T_{\alpha} \sqsubseteq H_{\alpha}$ such that $p^{k} G_{\alpha} \subseteq S_{\alpha}, p^{k} H_{\alpha} \subseteq T_{\alpha}$, and $S_{\alpha} \simeq T_{\alpha}$.

Corollary 4.16. Let $G$ and $H$ be direct sums of countable reduced p-groups. If $G \simeq H$ in $\mathscr{A} / \mathscr{B}$, then the corresponding Ulm factors of $G$ and $H$ are uniformiy quasi-isomorphic.

Proof. Apply 4.13 to see that the corresponding Ulm factors of $G$ and $H$ satisfy (I) with $k$ fixed. Now apply 4.2.

Lemma 4.17. Let $G$ be a reduced p-group. Then $(G / A)^{\beta}=G^{\beta} / A$ for all $\beta \leqq \alpha$ whenever $A \subseteq G^{\alpha}$.

Proof. The proof is by induction on $\beta$. For $\beta=0$, the lemma is clear. Suppose $\beta=1$ and let $x+A \in(G / A)^{1}$. Then $x+A=p^{n} x_{n}+A$ for each $n \geqq 0$. So $x=p^{n} x_{n}+a_{n} \in p^{n} G$ for each $n \geqq 0$. Thus $x+$ $A \in G^{1} / A$. Now let $\beta>1$ and assume $(G / A)^{\gamma}=G^{\prime} / A$ for all $\gamma<\beta$. If $\beta=\gamma+1$, then $(G / A)^{\beta}=\left(G^{\gamma} / A\right)^{1}=G^{\gamma+1} / A=G^{3} / A$. Suppose $\beta$ is a limit ordinal. Then

$$
(G / A)^{\beta}=\bigcap_{\gamma<\beta}(G / A)^{\gamma}=\bigcap_{\gamma<\beta}\left(G^{\gamma} / A\right)=\left(\bigcap_{\gamma<\beta} G^{\gamma}\right) / A=G^{\beta} / A .
$$

The proof is complete.

Lemma 4.18. Let $G$ be a reduced p-group and $k \geqq 0$. Then $\left(G / G^{\alpha}\left[p^{k}\right]\right)^{\beta}=G^{\beta} / G^{\alpha}\left[p^{k}\right]$ for $\beta \leqq \alpha$ and $\left(G / G^{\alpha}\left[p^{k}\right]\right)^{\alpha} \simeq p^{k} G^{\alpha}$.

Proof. The lemma follows from 4.17.

CoRollary 4.19. Let $G$ be a reduced p-group, $\alpha$ an ordinal, and $k \geqq 0$. Then $G / G^{\alpha}\left[p^{k}\right]$ has Ulm factors $G^{\beta} / G^{\beta+1}$ for $\beta<\alpha, p^{k} G^{\alpha} / G^{\alpha+1}$, and $G^{\beta} / G^{\beta+1}$ for $\beta>\alpha$. 
Proof. Let $\beta<\alpha$. Then $\beta+1 \leqq \alpha$ and by 4.17 ,

$$
\left(G / G^{\alpha}\left[p^{k}\right]\right)^{\beta} /\left(G / G^{\alpha}\left[p^{k}\right]\right)^{\beta+1}=\left(G^{\beta} / G^{\alpha}\left[p^{k}\right]\right) /\left(G^{\beta+1} / G^{\alpha}\left[p^{k}\right]\right) \simeq G^{\beta} / G^{\beta+1} .
$$

By 4.18 and since $X^{\alpha} / X^{\alpha+1} \simeq Y^{\alpha} / Y^{\alpha+1}$ whenever $X$ and $Y$ are isomorphic $p$-groups, $\left(G / G^{\alpha}\left[p^{k}\right]\right)^{\alpha} /\left(G / G^{\alpha}\left[p^{k}\right]\right)^{\alpha+1} \simeq p^{k} G^{\alpha} / G^{\alpha+1}$. Furthermore, if $\beta>\alpha$, say $\beta=\alpha+\delta$, then

$$
\begin{aligned}
\left(G / G^{\alpha}\left[p^{k}\right]\right)^{\beta} /\left(G / G^{\alpha}\left[p^{k}\right]\right)^{\beta+1} & \simeq\left(G^{\alpha} / G^{\alpha}\left[p^{k}\right]\right)^{\delta} /\left(G^{\alpha} / G^{\alpha}\left[p^{k}\right]\right)^{\delta+1} \\
& \simeq\left(p^{k} G^{\alpha}\right)^{\delta} /\left(p^{k} G^{\alpha}\right)^{\delta+1}=G^{\alpha+\delta} / G^{\alpha+\delta+1}=G^{\beta} / G^{\beta+1} .
\end{aligned}
$$

LEMMA 4.20. Let $G$ be a countable reduced p-group of length $\tau$ and with Ulm factors $G_{\alpha}$. For each $\alpha<\tau$, let $G_{\alpha}=S_{\alpha} \oplus T_{\alpha}$ where both $S_{\alpha}$ and $T_{\alpha}$ are unbounded whenever $G_{\alpha}$ is. Let $H$ be a countable reduced p-group of length $\tau$ and with Ulm factors $p^{n_{\alpha}} S_{\alpha} \oplus T_{\alpha}$ where $0 \leqq n_{\alpha} \leqq k, k$ fixed. (Such a group exists by Zippin's theorem.) Then $G / A \simeq H$ for some $A \subseteq G\left[p^{k}\right]$.

Proof. For $\tau=0$, there is nothing to prove. If $\tau=1, G$ is a direct sum of cyclic groups and the lemma is clear. Assume $\tau>1$. For the present, we will assume $G_{\tau-1}$ is unbounded if $\tau-1$ exists. For each $\alpha<\tau$, let $T_{\alpha}=\sum_{\beta<\tau} G_{\alpha \beta}$ where $G_{\alpha \beta}$ is unbounded for $\beta \neq \alpha$ and $G_{\alpha \alpha}=0$. For each $\alpha<\tau$, let $K_{\alpha}$ and $L_{\alpha}$ be countable reduced $p$-groups of length $\tau$ whose $\alpha$ th Ulm factors are $p^{n}{ }^{n} S_{\alpha}$ and $S_{\alpha}$, respectively, and whose $\beta$ th Ulm factors, $\beta \neq \alpha$, are $G_{\beta \alpha}$. Now $G \simeq \sum_{\alpha<\tau} L_{\alpha}$ and applying 4.18, $L_{\alpha} / L_{\alpha}^{\alpha}\left[p^{n} \alpha\right] \simeq K_{\alpha}$ for each $\alpha<\tau$. Let $K=\sum_{\alpha<\tau} K_{\alpha}$. Then $G / A \simeq K$ where $A=\sum_{\alpha<\tau} L_{\alpha}^{\alpha}\left[p^{n} \alpha\right]$. Moreover, $K$ has Ulm factors $p^{n}{ }^{n} S_{\alpha} \oplus T_{\alpha}$. Hence $G / A \simeq K \simeq H$.

Now assume $\tau-1$ exists and $G_{\tau-1}$ is bounded. For $\alpha<\tau-1$, Let $T_{\alpha}$ be as above. Let $K_{0}$ be a countable reduced $p$-group whose Ulm factors are $p^{n_{0}} S_{0}, G_{\beta_{0}}$ for $0<\beta<\tau-1$, and $T_{\tau-1}$. Let $L_{0}$ be a countable reduced $p$-group whose Ulm factors are $S_{0}, G_{\beta_{0}}$ for $0<\beta<\tau-1$, and $T_{\tau-1}$. For $0<\alpha<\tau-1$, let $K_{\alpha}$ and $L_{\alpha}$ be countable reduced $p$-groups of length $\tau-1$ whose $\alpha$ th Ulm factors are $p^{n}{ }_{\alpha} S_{\alpha}$ and $S_{\alpha}$, respectively, and whose $\beta$ th Ulm factors, $\beta \neq \alpha$, are $G_{\beta \alpha}$. Let $K_{\tau-1}$ and $L_{\tau-1}$ be countable reduced $p$-groups whose Ulm factors are $G_{\beta, \tau-1}$ for $0 \leqq \beta<\tau-1$ and whose $(\tau-1)$ st Ulm factors are $p^{n_{\tau-1}} S_{\tau-1}$ and $S_{\tau-1}$, respectively. Let $K=\sum_{\alpha<\tau} K_{\alpha}$ and $A=$ $\sum_{\alpha<\tau} L_{\alpha}^{\alpha}\left[p^{n} \alpha\right]$. As above $G / A \simeq K \simeq H$. This completes the proof.

Lemma 4.21. Let $G$ be as in 4.20. For each $\alpha<\tau$, let $G_{\alpha}=$ $S_{\alpha} \oplus T_{\alpha}$. Let $H$ be a countable reduced p-group with Ulm factors $p^{n} S_{\alpha} \oplus T_{\alpha}, n \geqq 0$. Then $G \simeq H$ in $\mathscr{A} / \mathscr{B}$. In fact, there is an epimorphism $f: G \rightarrow p^{n} H$ such that $\operatorname{Ker} f$ is bounded by $p^{3 n}$. 
Proof. Let $I_{1}=\left\{\alpha<\tau \mid S_{\alpha}\right.$ and $T_{\alpha}$ are both unbounded $\}, I_{2}=\left\{\alpha<\tau \mid S_{\alpha}\right.$ is unbounded and $T_{\alpha}$ is bounded $\}, I_{3}=\left\{\alpha<\tau \mid S_{\alpha}\right.$ is bounded and $T_{\alpha}$ is unbounded, and $I_{4}=\{\tau-1\}$ if $\tau-1$ exists and $G_{\tau-1}$ is bounded, otherwise $I_{4}=\varnothing$. If $\alpha \in I_{2}$, let $S_{\alpha}=S_{\alpha}^{\prime} \oplus S_{\alpha}^{\prime \prime}$ where both $S_{\alpha}^{\prime}$ and $S_{\alpha}^{\prime \prime}$ are unbounded. If $\alpha \in I_{3}$, let $T_{\alpha}=T_{\alpha}^{\prime} \oplus T_{\alpha}^{\prime \prime}$ where both $T_{\alpha}^{\prime}$ and $T_{\alpha}^{\prime \prime}$ are unbounded. Let $K$ have Ulm factors as follows:

$$
\begin{aligned}
& p^{n} S_{\alpha} \oplus T_{\alpha} \text { if } \alpha \in I_{1} \cap I_{4}, \\
& p^{n} S_{\alpha}^{\prime} \oplus S_{\alpha}^{\prime \prime} \oplus T_{\alpha}=P^{n} S_{\alpha} \oplus T_{\alpha} \text { if } \alpha \in I_{2}, \\
& p^{n} S_{\alpha} \oplus p^{n} T_{\alpha}^{\prime} \oplus T_{\alpha}^{\prime \prime} \text { if } \alpha \in I_{3} .
\end{aligned}
$$

By $4.20, G / A \simeq K$ for some $A \subseteq G\left[p^{n}\right]$. Let $L$ have Ulm factors as follows:

$$
\begin{aligned}
& p^{n} S_{\alpha} \oplus T_{\alpha} \text { if } \alpha \in I_{1} \cap I_{4}, \\
& p^{n} S_{\alpha}^{\prime} \oplus p^{n} S_{\alpha}^{\prime \prime} \oplus T_{\alpha}=p^{n} S_{\alpha} \oplus T_{\alpha} \text { if } \alpha \in I_{2}, \\
& p^{n} S_{\alpha} \oplus p^{n} T_{\alpha}^{\prime} \oplus T_{\alpha}^{\prime \prime} \text { if } \alpha \in I_{3} .
\end{aligned}
$$

By $4.20, K / B \simeq L$ for some $B \subseteq G\left[p^{n}\right]$. Let $H$ have Ulm factors as follows:

$$
\begin{aligned}
& p^{n} S_{\alpha} \oplus T_{\alpha} \text { if } \alpha \in I_{1} \cap I_{2} \cap I_{4}, \\
& p^{n} S_{\alpha} \oplus T_{\alpha}^{\prime} \oplus T_{\alpha}^{\prime \prime}=p^{n} S_{\alpha} \oplus T_{\alpha} \text { if } \alpha \in I_{3} .
\end{aligned}
$$

By $4.20, H / C \simeq L$ for some $C \subseteq H\left[p^{n}\right]$. Hence $G \simeq H$ in $\mathscr{A} / \mathscr{B}$. Let $f$ be the composition $G \rightarrow G / A \simeq K \rightarrow K / B \simeq L \simeq H / C \rightarrow H / H\left[p^{n}\right] \simeq$ $p^{n} H$. The lemma follows.

LEMMA 4.22. Let $G$ be a direct sum of countable reduced $p$ groups, $n \geqq 0$, and suppose $G$ has length $\tau$. Let $G$ have Ulm factors $G_{\alpha}$. For each $\alpha<\tau$, let $G_{\alpha}=S_{\alpha} \oplus T_{\alpha}$. Then there is a p-group $H$ such that

(i) $H$ is a direct sum of countable reduced groups,

(ii) $H$ has Ulm factors $p^{n} S_{\alpha} \oplus T_{\alpha}$,

(iii) $G \simeq H$ in $\mathscr{A} / \mathscr{B}$.

Proof. Let $G=\sum_{\lambda \in_{\Lambda}} X_{\lambda}$ where $\left|X_{\lambda}\right| \leqq \boldsymbol{\aleph}_{0}$ for each $\lambda \in \Lambda$. Let $\tau_{\lambda}$ be the length of $X_{\lambda}$ and $X_{\lambda \alpha}$ its $\alpha$ th Ulm factor. For $\tau_{\lambda} \leqq \alpha<\tau, X_{\lambda \alpha}=0$. Now $G_{\alpha}=\sum_{i \in \Lambda} X_{\lambda \alpha}$ for each $\alpha<\tau$. Let $X_{\lambda \alpha}^{B}, S_{\alpha}^{B}$, and $T_{\alpha}^{B}$ be bases of $X_{\lambda \alpha}, S_{\alpha}$, and $T_{\alpha}$, respectively. For each $\alpha<\tau$, there is an equivalence $\pi_{\alpha}: \bigcup_{\lambda \in \Lambda} X_{\lambda \alpha}^{B} \rightarrow S_{\alpha}^{B} \cup T_{\alpha}^{B}$ which preserves exponents. For $\lambda \in \Lambda$ and $\alpha<\tau_{\lambda}$, define

$$
\begin{aligned}
& S_{\lambda \alpha}=\sum\left\{\langle a\rangle \mid a \in X_{\lambda \alpha}^{B}, \pi_{\alpha}(a) \in S_{\alpha}^{B}\right\}, \\
& T_{\lambda \alpha}=\sum\left\{\langle a\rangle \mid a \in X_{\lambda \alpha}^{B}, \pi_{\alpha}(a) \in T_{\alpha}^{B}\right\} .
\end{aligned}
$$


If $\tau_{\lambda} \leqq \alpha<\tau$, let $S_{\lambda \alpha}=T_{\lambda \alpha}=0$. Then $X_{\lambda \alpha}=S_{\lambda \alpha} \oplus T_{\lambda \alpha}, \sum_{\lambda \in \Lambda} S_{\lambda \alpha} \simeq S_{\alpha}$, and $\sum_{\lambda \epsilon_{A}} T_{\lambda \alpha} \simeq T_{\alpha}$. Let $Y_{\lambda \alpha}=p^{n} S_{\lambda \alpha} \oplus T_{\lambda \alpha}$. Then $Y_{\lambda \alpha}$ is countable and if $\alpha<\tau_{\lambda}, Y_{\lambda \alpha}$ is unbounded with the possible exception of $Y_{\lambda, \tau_{\lambda-1}}$ if $\tau_{\lambda}-1$ exists. Let $Y_{\lambda}$ be a countable reduced $p$-group of length $\tau_{\lambda}$ and with Ulm factors $Y_{2, \alpha}$. By 4.20, there is an epimorphism $f_{\lambda}: X_{\lambda} \rightarrow p^{n} Y_{\lambda}$ such that $p^{3 n} \operatorname{Ker} f=0$. Let $H=\sum_{\lambda_{1}} Y_{\lambda}$. Then there is an epimorphism $f: G \rightarrow p^{n} H$ such that $p^{3 n} \operatorname{Ker} f=0$. Hence $G \simeq H$ in $\mathscr{A} / \mathscr{B}$. Clearly $H$ is a direct sum of countable groups with Ulm factors $p^{n} S_{\alpha} \oplus T_{\alpha}$.

Lemma 4.23. Let $G$ be a direct sum of countable reduced pgroups. Let $G$ have length $\tau$ and Ulm factors $G_{\alpha}$. Then there is a reduced p-group $H$ such that

(i) $H$ is a direct sum of countable groups,

(ii) $H$ has Ulm factors $G_{\alpha} \oplus \sum_{\left|G_{\alpha}\right| \aleph_{0}} C(p), \alpha<\tau$,

(iii) $G \simeq H$ in $\mathscr{A} / \mathscr{S}$.

Proof. Write $G=\sum_{\lambda \epsilon_{\Lambda}} X_{\lambda}$ where $\left|X_{\lambda}\right| \leqq \aleph_{0}$ for each $\lambda \in \Lambda$. Let $\tau_{\lambda}$ be the length of $X_{\lambda}$ and $X_{\lambda \alpha}$ its $\alpha$ th Ulm factor. Then $G_{\alpha}=\sum_{\lambda \epsilon_{\Lambda}} X_{\lambda \alpha}$. For $\lambda \in \Lambda$ and $\alpha<\tau_{\lambda}$, let $Y_{\lambda \alpha} \simeq X_{\lambda \alpha} \oplus \sum_{\aleph_{0}} C(p)$. If $\tau_{\lambda} \leqq \alpha<\tau$, let $Y_{\lambda \alpha}=0$. Let $Y_{\lambda}$ be the countable reduced $p$-group of length $\tau_{\lambda}$ and with Ulm factors $Y_{\lambda \alpha}, \alpha<\tau_{\lambda}$. For $\alpha<\tau$, let $\Lambda_{\alpha}=\left\{\lambda \in \Lambda \mid X_{\lambda \alpha} \neq 0\right\}$. Then $\left|G_{\alpha}\right| \boldsymbol{\aleph}_{0}=\left|\Lambda_{\alpha}\right| \boldsymbol{\aleph}_{0}$ for $\alpha<\tau$. Let $H=\sum_{\lambda \in_{\Lambda}} Y_{\lambda}$. Then $H$ is a direct sum of countable reduced $p$-groups whose $\alpha$ th Ulm factor is

$$
\begin{aligned}
H_{\alpha} & =\sum_{\lambda \epsilon_{A}} Y_{\lambda \alpha} \simeq \sum_{\lambda \epsilon_{A}}\left(X_{\lambda \alpha} \oplus \sum_{\aleph_{0}} C(p)\right) \\
& =\sum_{\lambda \epsilon_{1}} X_{\lambda \alpha} \oplus \sum_{i \in 1} \sum_{\boldsymbol{\aleph}_{0}} C(p)=G_{\alpha} \oplus \sum_{\left|G_{\alpha}\right|} \sum_{\boldsymbol{\aleph}_{0}} C(p) .
\end{aligned}
$$

By 4.22, $H$ is isomorphic in $\mathscr{A} / \mathscr{S}$ to the direct sum of countable groups whose Ulm factors are $G_{\alpha} \oplus p\left(\sum_{\left|G_{\alpha}\right|} \sum_{\aleph_{0}} C(p)\right)=G_{\alpha}$; namely, $H \simeq G$ in $\mathscr{A} / \mathscr{B}$.

Lemma 4.24. Let $G$ and $H$ be direct sums of countable reduced p-groups whose corresponding Ulm factors are uniformly quasiisomorphic. Let $G$ and $H$ have the same length $\tau$ and Ulm factors $G_{\alpha}$ and $H_{\alpha}$, respectively. Suppose $f_{G_{\alpha}}(0)=\left|G_{\alpha}\right| \geqq \boldsymbol{\aleph}_{0}$ and $f_{I I_{\alpha}}(0)=$ $\left|H_{\alpha}\right| \geqq \aleph_{0}$ for each $\alpha<\tau$. Then there exists direct sums of countable reduced p-groups $K$ and $L$ with Ulm factors $K_{\alpha}$ and $L_{\alpha}$, respectively, such that

(i) $f_{K_{\alpha}}(0)=\left|K_{\alpha}\right|\left|L_{\alpha}\right| \boldsymbol{\aleph}_{0}=f_{L_{\alpha}}(0)$ for each $\alpha<\tau$,

(ii) $G \simeq K$ in $\mathscr{A} / \mathscr{B S}$,

(iii) $H \simeq L$ in $\mathscr{A} / \mathscr{B}$.

Proof. Let $I_{1}=\left\{\alpha<\tau|| G_{\alpha}|=| H_{\alpha} \mid\right\}, I_{2}=\left\{\alpha<\tau|| G_{\alpha}|<| H_{\alpha} \mid\right\}$, and $I_{3}=\left\{\alpha<\tau|| H_{\alpha}|<| G_{\alpha} \mid\right\}$. There is an integer $k \geqq 0$ and for 
each $\alpha<\tau$, subgroups $S_{\alpha} \subseteq G_{\alpha}$ and $T_{\alpha} \subseteq H_{\alpha}$ such that $p^{k} G_{\alpha} \subseteq S_{\alpha}$, $p^{k} H_{\alpha} \cong T_{\alpha}$, and $S_{\alpha} \simeq T_{\alpha}$. Thus $\left|H_{\alpha}<\right| G_{\alpha}|\geqq| p^{2 k} H_{\alpha} \mid$ for $\alpha \in I_{2}$ and $\left|G_{\alpha}\right|>\left|H_{\alpha}\right| \geqq\left|p^{2 k} G_{\alpha}\right|$ for $\alpha \in I_{3}$. If $\alpha \in I_{2}$, write $H_{\alpha}=H_{\alpha}^{\prime} \oplus \sum_{\left|G_{\alpha}\right|} C(p)$. If $\alpha \in I_{3}$, write $G_{\alpha}=G_{\alpha}^{\prime} \oplus \sum_{\left|H_{\alpha}\right|} C(p)$. By 4.22 , there is a direct sum of countable reduced $p$-groups $K$ whose Ulm factors $K_{\alpha}$ are $G_{\alpha}$ for $\alpha \in I_{1} \cup I_{2}$ and $p^{2 k} G_{\alpha}^{\prime} \oplus \sum_{\left|H_{\alpha}\right|} C(p)$ for $\alpha \in I_{3}$ and such that $G \simeq K$ in $\mathscr{A} / \mathscr{B}$. Similarly, there is a direct sum of countable reduced $p$-groups $L$ whose Ulm factors $L_{\alpha}$ are $H_{\alpha}$ for $\alpha \in I_{1} \cup I_{3}$ and $p^{2 k} H_{\alpha}^{\prime} \oplus \sum_{\left|G_{\alpha}\right|} C(p)$ for $\alpha \in I_{2}$ and such that $L \simeq H$ in $\mathscr{A} / \mathscr{B}$. For $\alpha \in I_{1} \cup I_{2}, f_{K_{\alpha}}(0)=$ $\left|G_{\alpha}\right|=\left|K_{\alpha}\right|$. For

$$
\begin{aligned}
\alpha \in I_{3},\left|H_{\alpha}\right| & \geqq f_{K_{\alpha}}(0) \leqq\left|K_{\alpha}\right| \leqq\left|p^{2 k} G_{\alpha}^{\prime}\right|+\left|H_{\alpha}\right| \\
& \leqq\left|G_{\alpha}\right|+\left|H_{\alpha}\right| \leqq\left|H_{\alpha}\right|+\left|H_{\alpha}\right| .
\end{aligned}
$$

By hypothesis, $\left|G_{\alpha}\right| \geqq \aleph_{0}$ and $\left|H_{\alpha}\right| \geqq \aleph_{0}$. Thus $\left|K_{\alpha}\right|=f_{K_{\alpha}}(0)=$ $\min \left(\left|G_{\alpha}\right|,\left|H_{\alpha}\right|\right)$ for $\alpha<\tau$. Likewise, $\left|L_{\alpha}\right|=f_{L_{\alpha}}(0)=\min \left(\left|G_{\alpha}\right|,\left|H_{\alpha}\right|\right)$ for $\alpha<\tau$. It now follows that $f_{K_{\alpha}}(0)=\left|K_{\alpha}\right|\left|L_{\alpha}\right| \boldsymbol{\aleph}_{0}=f_{L_{\alpha}}(0)$ for $\alpha<\tau$.

THEOREM 4.25. Let $G$ and $H$ be direct sums of countable reduced p-groups. Then $G \simeq H$ in $\mathscr{A} / \mathscr{B}$ if and only if their corresponding Ulm factors are uniformly quasi-isomorphic.

Proof. Assume that the corresponding Ulm factors of $G$ and $H$ are uniformly quasi-isomorphic. Let $G_{\alpha}$ and $H_{\alpha}$ be the $\alpha$ th Ulm factors of $G$ and $H$, respectively. If $G$ has length $\tau$ and $H$ has length $\tau+1$, then $H_{\tau}$ is bounded, $H / H_{\tau}$ is a direct sum of countable groups, and $G \simeq H$ in $\mathscr{A} / \mathscr{S}$ if and only if $G \simeq H / H_{\tau}$ in $\mathscr{A} / \mathscr{D}$. Moreover, the corresponding Ulm factors of $G$ and $H / H_{\tau}$ are uniformly quasi-isomorphic. Hence we may as well assume that $G$ and $H$ have the same length $\tau$. By 4.23, there exist direct sums of countable reduced $p$-groups $K$ and $L$ such that $K$ has Ulm factors

$$
G_{\alpha} \oplus \sum_{\left|\sigma_{0}\right|} \sum_{\mathbf{\aleph}_{0}} C(p)
$$

$L$ has Ulm factors $H_{\alpha} \oplus \sum_{\left|I I_{\alpha}\right|} \sum_{\boldsymbol{N}_{0}} C(p), G \simeq K$ in $\mathscr{C} / \mathscr{B}$, and $H \simeq L$ in $/ \mathscr{R}$. By 4.16, the corresponding Ulm factors of $G$ and $K(H$ and $L$ ) are uniformly quasi-isomorphic. In particular the corresponding Ulm factors of $K$ and $L$ are uniformly quasi-isomorphic. Moreover, $G \simeq H$ in $\mathscr{C} / \mathscr{B}$ if and only if $K \simeq L$ in $\mathscr{C} / \mathscr{B}$. Hence we may as well assume that $f_{G_{\alpha}}(0)=\left|G_{\alpha}\right| \boldsymbol{\aleph}_{0}$ and $f_{H_{\alpha}}(0)=\left|H_{\alpha}\right| \boldsymbol{\aleph}_{0}$ for each $\alpha<\tau$. By 4.24, we may likewise assume that $f_{\sigma_{\alpha}}(0)=\left|G_{\alpha}\right|\left|H_{\alpha}\right| \boldsymbol{\aleph}_{0}=f_{H_{a}}(0)$ for each $\alpha<\tau$. There is an integer $k \geqq 0$ and for each $\alpha<\tau$, subgroups $S_{\alpha} \subseteq G_{\alpha}$ and $T_{\alpha} \subseteq H_{\alpha}$ such that $p^{k} G_{\alpha} \subseteq S_{\alpha}, p^{k} H_{\alpha} \subseteq T_{\alpha}$ and $S_{\alpha} \simeq T_{\alpha}$. Thus for each $\alpha<\tau$ and all integers $n \geqq 0$ and $r \geqq 0$ 


$$
\sum_{j=0}^{r} f_{C}(n+k+j) \leqq \sum_{j=0}^{r+2 k} f_{H}(n+j)
$$

and

$$
\sum_{j=0}^{r} f_{H}(n+k+j) \leqq \sum_{j=0}^{r+2 k} f_{G}(n+j)
$$

(For a proof of this fact, see [1].) Now by 4.1, we can write $G_{\alpha}=G_{\alpha, 0} \oplus \cdots \oplus G_{\alpha, 2 k}$ and $H_{\alpha}=H_{\alpha, 0} \oplus \cdots \oplus H_{\alpha, 2 k}$ where $p^{k} G_{\alpha, 0} \simeq$ $H_{\alpha, 0}, p^{k-1} G_{\alpha, 1} \simeq H_{\alpha, 1}, \cdots, G_{\alpha, k} \simeq H_{\alpha, k}, G_{\alpha, k+1} \simeq p H_{\alpha, k+1}, \cdots, G_{\alpha, 2 k} \simeq p^{k} H_{\alpha, 2 k}$ for each $\alpha<\tau$. Now apply 4.22 as needed.

THEOREM 4.26. Let $G$ and $H$ be direct sums of countable reduced p-groups. Then $G \simeq H$ in $\mathscr{A} / \mathscr{B}$ if and only if there is an integer $k \geqq 0$ such that for all ordinals $\alpha$ and all integers $r \geqq 0$

$$
\sum_{j=0}^{r} f_{G}(\alpha+k+j) \leqq \sum_{j=0}^{r+2 k} f_{H}(\alpha+j)
$$

and

$$
\sum_{j=0}^{r} f_{H}(\alpha+k+j) \leqq \sum_{j=0}^{r+2 k} f_{G}(\alpha+j)
$$

Proof. Suppose the inequalities hold. Let $G_{\alpha}$ and $H_{\alpha}$ be the $\alpha$ th Ulm factors of $G$ and $H$, respectively. Then for each ordinal $\alpha$ and $r \leqq 0$

$$
\begin{aligned}
\sum_{j=0}^{r} f_{G_{\alpha}}(n+k+j) & =\sum_{j=0}^{r} f_{G}(\omega \alpha+n+k+j) \\
& \leqq \sum_{j=0}^{r+2 k} f_{H}(\omega \alpha+n+j)=\sum_{j=0}^{r+2 k} f_{H \alpha}(n+j)
\end{aligned}
$$

and similarly

$$
\sum_{j=0}^{r} f_{H_{\alpha}}(n+k+j) \leqq \sum_{j=0}^{r+2 k} f_{G_{\alpha}}(n+j)
$$

Now apply 4.2 to see that the corresponding Ulm factors of $G$ and $H$ are uniformly quasi-isomorphic. By 4.25, $G \simeq H$ in $\mathscr{A} / \mathscr{B}$.

The converse is 4.13 .

\section{REFERENCES}

1. R. A. Beaumont and R. S. Pierce, Quasi-isomorphism of p-groups, Proceedings of the colloquim on Abelian groups, ed. by L. Fuchs and E. T. Schmidt, Budapest, 1964, $13-27$.

2. Quasi-isomorphism of direct sums of cyclic groups, Acta Math. Sci. Hung. 16 (1965), 33-36. 
3. D. O. Cutler, Quasi-isomorphism for infinite Ahelian p-groups, Pacific J. Math. 16 (1966), 25-45.

4. L. Fuchs, Abelian Groups, Publishing House of the Hungarian Academy of Sciences, Budapest, 1958.

5. P. Gabriel, Des categories abeliennes, Bull. Soc. Math. France 90 (1962), 323-448.

6. A. Grothendieck, Surquelques points d'Algebra homologique, Tohoku Math. J. 9 (1957), 119-221.

7. P. Hill, Sums of countable primary groups (to appear)

8. - Quasi-isomorphism of primary groups (to appear)

9. J. M. Irwin and F. Richman, Direct sums of countable groups and related concepts, J. Algebra, 4 (1965), 443-450.

10. J. M. Irwin, C. Walker and E. A. Walker, On p-pure sequences of Abelian groups, Topics in Abelian groups, ed. by J. M. Irwin and E. A. Walker, Scott Foresman and Co., Chicago, 1963, 69-119.

11. B. Jónsson, On direct decompositions of torsion-free Abelian groups, Math. Scand. 7 (1959), 361-371.

12. G. Kolettis, Direct sums of countable groups, Duke Math. J. 27 (1960), 111-125.

13. S. MacLane, Homology, Springer-Verlag, Berlin, 1963.

14. E. A. Walker, Quotient categories and quasi-isomorphisms of Abelian groups, Proceedings of the colloquim on Abelian groups, ed. by L. Fuchs and E. T. Schmidt, Budapest, 1964, 147-163.

15. - On n-extensions of Abelian groups, Ann. Univ. Sci. Budapest. Eötvös Sect. Math. 8 (1965), 71-74.

Received December 13, 1966. The results of this paper were part of a doctor's thesis completed in August 1966 under Professor Elbert A. Walker at New Mexico State University. The work was partially supported by NSF Grant GP 3581. 



\section{PACIFIC JOURNAL OF MATHEMATICS}

\section{EDITORS}

\section{H. ROYDEN}

Stanford University

Stanford, California

\section{J. P. Jans}

University of Washington

Seattle, Washington 98105

\section{J. DUGUNDJI}

Department of Mathematics

Rice University

Houston, Texas 77001

RICHARD ARENS

University of California

Los Angeles, California 90024

\section{ASSOCIATE EDITORS}
E. F. BeCKenbaCH
B. H. NeumanN
F. WOLF
K. YosIDA

\section{SUPPORTING INSTITUTIONS}

UNIVERSITY OF BRITISH COLUMBIA CALIFORNIA INSTITUTE OF TECHNOLOGY UNIVERSITY OF CALIFORNIA MONTANA STATE UNIVERSITY UNIVERSITY OF NEVADA NEW MEXICO STATE UNIVERSITY OREGON STATE UNIVERSITY UNIVERSITY OF OREGON OSAKA UNIVERSITY UNIVERSITY OF SOUTHERN CALIFORNIA
STANFORD UNIVERSITY UNIVERSITY OF TOKYO UNIVERSITY OF UTAH WASHINGTON STATE UNIVERSITY UNIVERSITY OF WASHINGTON

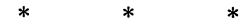

AMERICAN MATHEMATICAL SOCIETY CHEVRON RESEARCH CORPORATION TRW SYSTEMS

NAVAL ORDNANCE TEST STATION 


\section{Pacific Journal of Mathematics}

\section{Vol. 24, No. $1 \quad$ May, 1968}

Harry P. Allen, Lie algebras of type $D_{4}$ over algebraic number fields ...... 1

Charles Ballantine, Products of positive definite matrices. II............ 7

David W. Boyd, The spectral radius of averaging operators ............ 19

William Howard Caldwell, Hypercyclic rings ................... 29

Francis William Carroll, Some properties of sequences, with an application

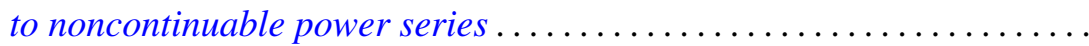

David Fleming Dawson, Matrix summability over certain classes of

sequences ordered with respect to rate of convergence ........... 51

D. W. Dubois, Second note on David Harrison's theory of preprimes. . . . . 57

Edgar Earle Enochs, A note on quasi-Frobenius rings.............. 69

Ronald J. Ensey, Isomorphism invariants for Abelian groups modulo bounded groups ................................ 71

Ronald Owen Fulp, Generalized semigroup kernels ................ 93

Bernard Robert Kripke and Richard Bruce Holmes, Interposition and approximation ................................. 103

Jack W. Macki and James Sai-Wing Wong, Oscillation of solutions to second-order nonlinear differential equations ..................

Lothrop Mittenthal, Operator valued analytic functions and generalizations

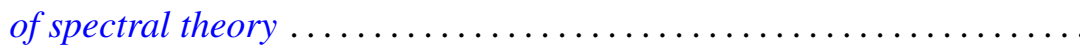

T. S. Motzkin and J. L. Walsh, A persistent local maximum of the pth power deviation on an interval, $p<1 \ldots \ldots \ldots \ldots \ldots \ldots \ldots \ldots \ldots \ldots . \ldots \ldots$

Jerome L. Paul, Sequences of homeomorphisms which converge to homeomorphisms ...........................

Maxwell Alexander Rosenlicht, Liouville's theorem on functions with elementary integrals.

Joseph Goeffrey Rosenstein, Initial segments of degrees .

$\mathrm{H}$. Subramanian, Ideal neighbourhoods in a ring ............

Dalton Tarwater, Galois cohomology of abelian groups . .

James Patrick Williams, Schwarz norms for operators ... .

Raymond Y. T. Wong, A wild Cantor set in the Hilbert cube. 